\title{
REAL-TIME RAINDROP DETECTION BASED ON DEEP LEARNING ALGORITHM
}

\author{
Florin Bogdan MARIN, Mihaela MARIN \\ "Dunarea de Jos" University of Galati, Romania \\ e-mail: florin.marin@ugal.ro
}

\begin{abstract}
The goal of this research is to develop an in-vehicle computerized system able to detect the raindrops on windshield and warn the driver and start the windscreen wiper in order to avoid that computer vision to acquire blurred images. This feature is important in order to develop Advanced Driver Assistance System based on computer vision. The system should be able specific scenarios that do not allow the ADAS computer vision feature to work properly. Rain drop detection will allow a more reliable Advanced Driver Assistance System.
\end{abstract}

KEYWORDS: computer vision, passenger fatigue detection, Advanced Driver Assistance System

\section{Introduction}

With more than 50000 casualties in Europe, Advanced Aided Driver Assistance (ADAS) systems solutions are rapidly becoming an essential feature of many car manufacturer in order to provide road safety. Besides many sensors used (lidar, radar, ultrasonic) computer vision is used mainly in most of ADAS systems such as obstacle detection, lane detection, pedestrian detection. Due to the variable environmental that cameras used in ADAS computer vision application must be used the developer must address additional challenges, such as corrupted data flow or failure of image acquisition. One of the issues is rain-drop detection. In this case, the system automatically starts windshield wipers and/or alert the driver that the system cannot be trusted due to the bad image. There are many attempts to detect weather conditions from videos or images for computer vision applications $[1,5,6]$. Raindrops can be described as transparent sphere reflecting light in a specific pattern to the camera. Also, the light is refracted to the camera leading to a distorting image and build a blurred image. Due to these facts the detection of raindrops can be detected.

Several solutions that are assisting the driver are commercially available or developed as proof of concept [2-4]. Dozens of ADAS features are taking into account traffic sign recognition, forward collision warning, lane warning departure, parking assistance, etc.
Self-driving vehicles, also named autonomous vehicles must also use computer vision processing besides several sensors in order to understand the environment to safely navigate without driver decisions. Consequently, autonomous vehicle needs to detect rain drops detections in order to take actions such as control de windscreen wiper or to inform the driver cannot longer operate in safety.

Real time raindrop detection is significant not only to the ADAS system but also for future autonomous vehicles. The development of such detection techniques is of paramount significance with respect to its contribution in the field of computer vision-based applications applied to ADAS. It is also important to be utilized by all computer vision based ADAS for keeping their feature reliable and ensure the windshield is clear and allows reliable images to be processed. The study will also be significant also for other real time surveillance applications such as security cameras, wild animal surveillance. The detection of raindrop will allow the system to inform the user or to activate windshield wiper [3, 5-7]. The detection of windshield wiper is also another objective for ADAS based computer vision systems, as it shouldn't process information when the wipers are in the scene.

Raindrops on windshields are within large range of shape and size. Due to the fact that position in space is quite short distance to the camera this translates in optic effect such as blur, bad focus of the cameras.

There are several approaches for raindrop detection such as Gaussians filter to detect snow and 
fog based on the dynamics of the spatial and temporal dimensions of images. This method is detecting in fact texture of the rain drops.

Computer vision programmers also imagined Canny Edge detection algorithm for detection of raindrops. Estimating the amount of raindrops is important in order to asses time to control the windshield wiper, taking into account the visibility.
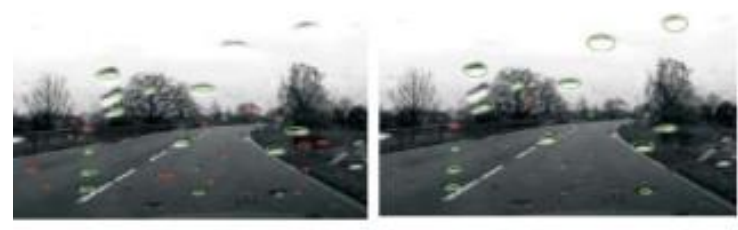

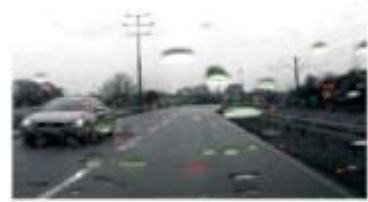

a. BLUR

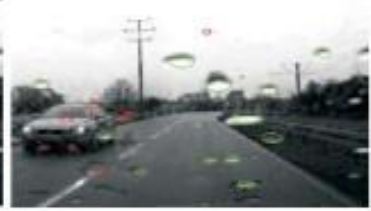

b. RIGSEC+BLUR
Fig. 1. Detection using BLUR and combination BLUR+RIGSEC. Red marks show False Positives [1]

Several approaches require settings for as camera optics but this method can only be applied in specific manufacturer hardware and not to the mobile phone application designed as ADAS platform.

Analyzing urban scene images while the sky has the same color as raindrops is a difficult task taking into account that the process must be performed real time and provide fast solutions concerning image registration. It seems that in these areas, the detection rate is low.

The important feature of the deep neural networks is that object detection is based on training the model using several images. In such a way the networks allow learning automatically from training data samples. A deep neural network [4] is I different from a classical neuronal network as it has at least one hidden middle layer (Fig. 2), besides inputs and output layers compared to a classical neural network that have an input and output layer. Each neuronal node forwards a weighted input which is calculated from previous layer. Deep neuronal network is a good solution for many computer vision applications. Light refraction of the raindrops causes color changes depending on the background color.

\section{Technique proposed}

Experimental results were achieved using a hardware platform made of webcam the Logitech
C170 placed on dashboard (Fig. 2), and as processing unit the Lenovo with an Intel ${ }^{\circledR}$ Core $^{\mathrm{TM}}$ i5-1035G1 processor. The video sequences were acquired at 20 frames per second. The proposed approach was implemented in Microsoft Visual $\mathrm{C}++$ and OPenCV and OPEN VINO libraries. Figure 2 shows general algorithm to detect drop rain. While preliminary tests have shown that the proposed approach works well in the all-weather environments, during test an accident occurred, the windshield was broken. This might occur at a smaller scale and is leading that ADAS computer vision-based features to fail. In this case the system should warn the driver that ADAS features are not reliable.

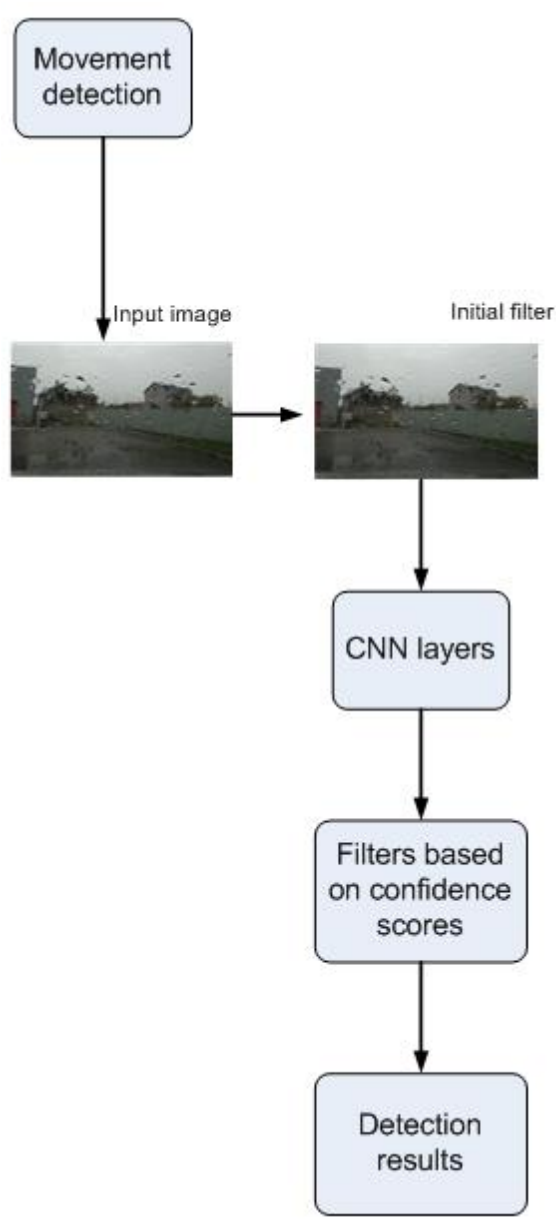
Fig. 2. General algorithm used for raindrop
detection

A model able of detecting realistic rain drop on glass surfaces demands small amount of dimensional parameterization in order to allow very low computational resources. The algorithm to identify rain (Fig. 3) drop is should run while others computer vision for ADAS are also working, same that might need complex calculations which result in serious computational cost. Moreover, running on the same 


\section{THE ANNALS OF "DUNAREA DE JOS" UNIVERSITY OF GALATI \\ FASCICLE IX. METALLURGY AND MATERIALS SCIENCE \\ $\mathrm{N}^{\circ} .4$ - 2020, ISSN 2668-4748; e-ISSN 2668-4756 \\ Article DOI: https://doi.org/10.35219/mms.2020.4.09}

processing unit of several algorithm to detect lane, obstacles, pedestrians, etc. will make the issue of computational resources important. The neuronal model should run not every frame, but also when the luminosity is changed to a low level, as most raindrop occurred when low luminosity occur, but also there are exceptions concerning weather conditions. In this respect the design parameters should be carefully tuned. CNNs are very efficient because they reuse a lot of weights transmitted across the network. OpenVino was used with Keras learning data in order to train with our data samples. The generic algorithm is described in Fig. 3. The movement detection filters images where motion is detected to some areas, whereas some areas are static. In such a way not all the images are processed in order to achieve a fast overall algorithm, taking into account that this rain drop detection is an auxiliary feature and not the main feature of a ADAS system. The input image is filtered in order to contrast contours with blurred image. The algorithm is tuned in such a way that in case of more than $20 \%$ of covering the windshield the system should run the windshield wipers. This percent should be at driver choice in order to achieve best comfort. CNN layers will identify several layers and will provide confidence score that is providing the

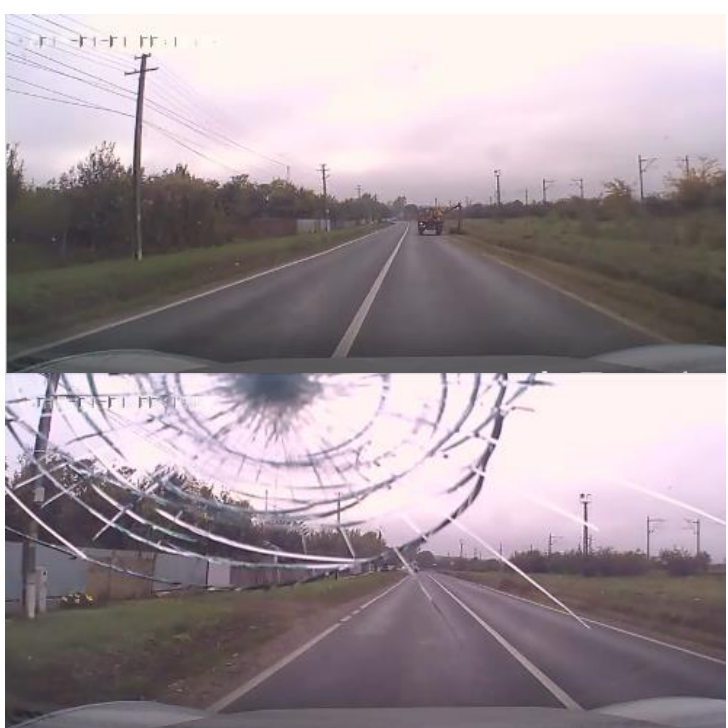

Fig. 3. Broken windshield

In case of the accident happened of broken windshield (Fig. 3), the system detected successfully that the image is not accurate in order to be processed. The developed algorithm is detecting most of the raindrops (Fig. 4). detection results.

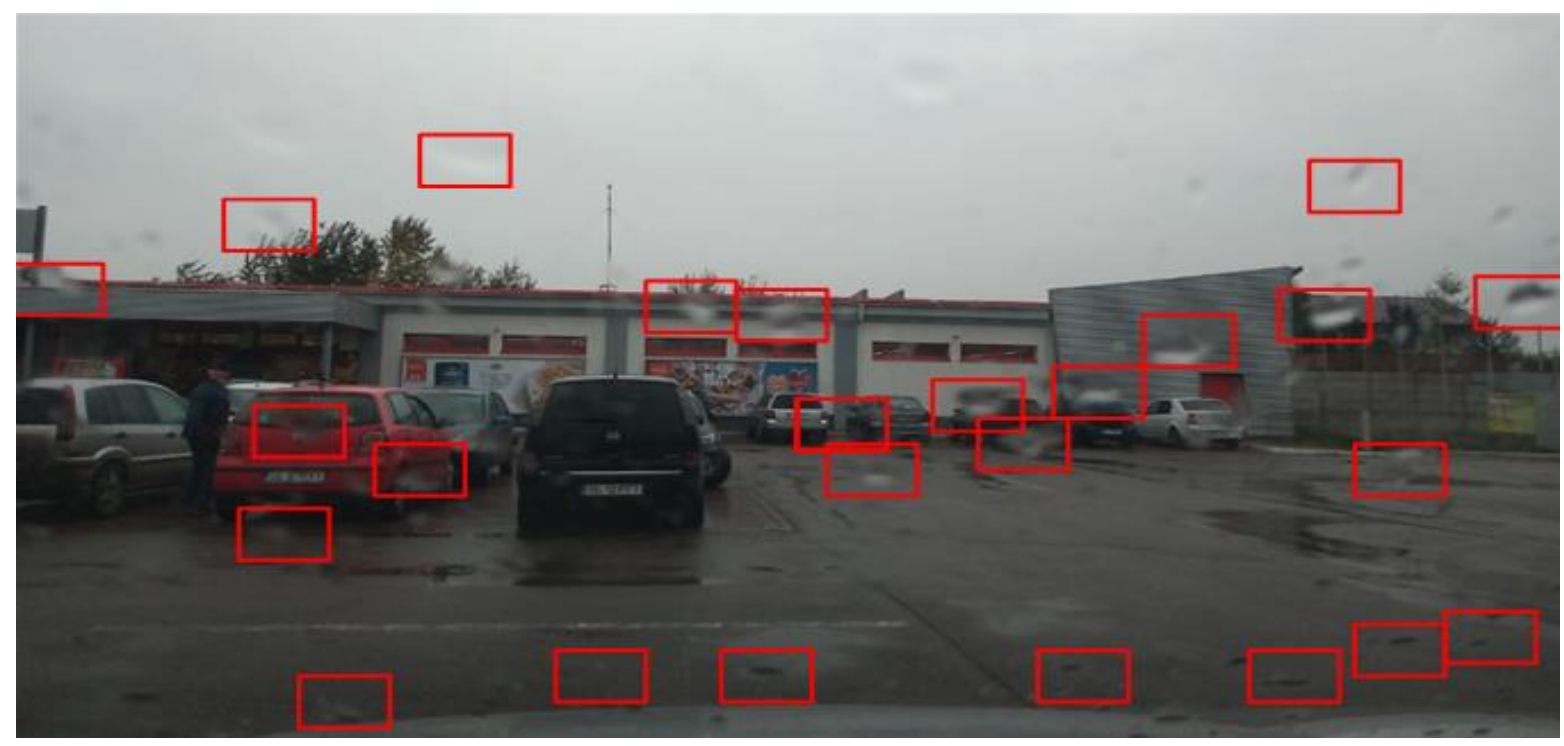

Fig. 4. Face detection and eye opened detection

Tested on 10 hours of videos, the algorithm shows a good performance with a rate of detection of $85 \%$ of the raindrops on the windshield. Due to the colors similarity we also counted $7 \%$ false positive detection (see Tab. 1).

Real-time performance on the processor mentioned indicate a fast algorithm, a frame
(1200X800) is processed in 100-200 ms depending on the image and raindrop coverage.

Tab 1. Statistics concerning feature detected

\begin{tabular}{|c|c|}
\hline No of video sequences & total $10 \mathrm{~h}$ \\
\hline Rate of detection & $85 \%$ \\
\hline False positive & $7 \%$ \\
\hline
\end{tabular}




\section{Conclusions}

The present study has shown the importance for ADAS system of the feature to detect raindrops in order to run the windshield wiper or to warn the driver. Although the method shows good potential in detecting raindrops from daytime images, given the fact that we use web camera and not night camera, the proposed method is limited to raindrops detection in constraint conditions such as daytime and requires further development to detect raindrops during the night.

\section{References}

[1]. Saba N. Karbhari, Pansambal B. H., Raindrop Detection: Performance analysis of recent approaches for the use in electronic driver assistance systems.
[2]. Shladover S. E., Review of the state of development of advanced vehicle control systems, Vehicle System Dynamics, 24(6-7), p. 551-595, 1995.

[3]. Aurelien Cord, Nicolas Gimonet, Detecting Unfocused Raindrops: In-Vehicle Multipurpose Cameras, Robotics \& Automation Magazine IEEE, vol. 21, no. 1, p. 49-56, 2014.

[4]. Jonathan Masci, Advances in Deep Learning for Vision, with Applications to Industrial Inspection Classification, Segmentation and Morphological extensions, Doctoral Dissertation submitted to the Faculty of Informatics of the Università della Svizzera Italiana. [5]. Roser M., Geiger A., Video-based raindrop detection for improved image registration, in Proc. IEEE Int. Conf. Computer Vision Workshops, p. 570-577, 2009.

[6]. Jee Hun Park, Man Ho Kim, Hong Jun Im, Kyung Chang Lee and Suk Lee, Development of Vision-Based Control Smart Windshield Wiper System for Intelligent Vehicle, SICE-ICASE International Joint Conference, 2006.

[7]. Fawazi Nashashibi, Raoul De Charette de La Contrie, Alexandre Lia, Detection of Unfocused Raindrops on a Windscreen using Low-Level Image Processing, International Conference on Control, Automation, Robotics and Vision: ICARV, Dec 2010. 